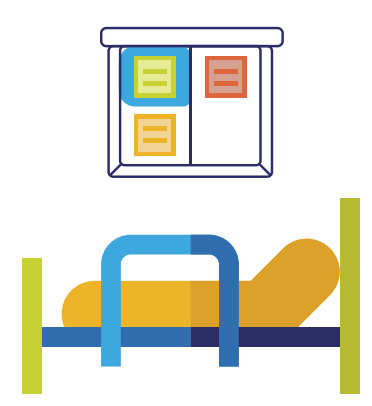

\title{
METODOLOGIA KANBAN COMO ESTRATÉGIA NA GESTÃO DE LEITOS NO HOSPITAL UNIVERSITÁRIO PROFESSOR EDGARD SANTOS - HUPES
}

\author{
METHODOLOGY KANBAN AS STRATEGY IN BED MANAGEMENT IN HOSPITAL \\ UNIVERSITY PROFESSOR EDGARD SANTOS - HUPES
}

\begin{abstract}
Ana Karina Lima Alves Cerdeira
Enfermeira, Mestranda Profissional em Gestão e Inovação em Saúde - UFRN; Especialista em Qualidade Internacional em Saúde e Segurança do Paciente - FIOCRUZ; Especialista em Auditoria dos Sistemas e Serviços de Saúde - UFBA; Gestora da Unidade de Gestão e Regulação de Leitos do Hospital Universitário Professor Edgard Santos - HUPES/EBSERH/UFBA. ana.kary.alves@hotmail.com

Kairon Ramon Sabino de Paiva

Analista de Sistemas, Mestrando Profissional em Gestão e Inovação em Saúde - UFRN; Especialista em Informática em saúde - UNIFESP. kairon@ufrn.edu.br.

\section{Luis Antônio Queiroz}

Analista de Tecnologia da informação do Hospital Universitário Professor Edgard Santos - HUPES/EBSERH/ UFBA; Especialista em Engenharia de Software - UniFacs; Especialista em Processos de Negócios pelo Instituto Alfa América. luis.queiroz@ebserh.gov.br.

\section{Valdira Gonzaga Rodrigues}

Enfermeira, Mestra em Organização e Gestão em Saúde - UFBA; Especialista em Administração Hospitalar - Faculdade São Camilo; Especialista em Gestão Pública - Ministério da Saúde; Especialista em Auditoria e Gestão em Serviços de Saúde - FDC; Gestora do Setor de Regulação e Avaliação em Saúde do Hospital Universitário Professor Edgard Santos - EBSERH/UFBA. valdirago@gmail.com.
\end{abstract}

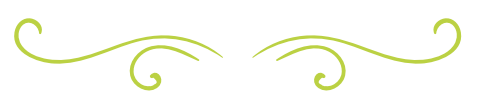

\section{RESUMO}

A superlotação hospitalar minimiza a qualidade da assistência, elevando os custos, a insatisfação dos usuários e profissionais, formando assim um ciclo vicioso. Esse é um problema mundial, que serve como desafio para os usuários do SUS e seus gestores. Nas organizações hospitalares, a metodologia Kanban está sendo adaptada como um instrumento para avaliação da qualidade assistencial, melhorias de fluxos e indicadores, sendo uma excelente ferramenta de 
apoio à gestão de leitos, permitindo otimização da oferta, redução de índice de permanência hospitalar, aumento da rotatividade dos leitos e de resolutividades no processo assistencial. $\bigcirc$ objetivo deste estudo é desenvolver um sistema para gestão de leitos do Hospital Universitário Professor Edgard Santos - HUPES a fim de monitorar o tempo de permanência do paciente, utilizando a metodologia Kanban como estratégia de trabalho. O método é uma pesquisa aplicada para um processo de desenvolvimento ágil do sistema baseado no OpenUP, compreendendo quatro fases: concepção, requisitos, elaboração e execução de forma interativa e cíclica, empregando-se o conceito arquitetural de camadas - MVC. Estabelece critérios de classificação por cores para a elaboração do painel de Monitoramento - vermelho, amarelo ou verde, conforme a metodologia Kanban -, calculando os dias de permanência do paciente na internação, direcionados pela competência e código SIGTAP, informado na Autorização de Internamento Hospitalar - AlH. Como resultado, a implementação desse método em conjunto com o sistema, qualificou o gerenciamento do cuidado, monitorando os pacientes internados nas unidades assistenciais, permitindo e descrevendo as características da complexidade quanto ao tempo de permanência, para auxiliar na gestão dos leitos e na produção de indicadores de cada unidade. Como conclusão, identificou-se o tempo de uso dos leitos ocupados, avaliação da qualidade da assistência, o que favorece os indicadores administrativos e de qualidade para o monitoramento do processo de internamento, proporcionando com isso a desospitalização.

Palavras-chave: Hospital Universitário. Gestão de Leitos. Sistema de Informação. Kanban.

\section{ABSTRACT}

Hospital overcrowding minimizes the quality of care, raising costs, dissatisfaction of users and professionals, forming a vicious cycle. This is a worldwide problem that serves as a challenge for SUS users and their managers. In hospital organizations, the Kanban methodology is being adapted as an instrument to assess the quality of care, improvements in flows and indicators, and is anexcellenttooltosupportbedmanagement, allowing optimization of supply, reduction of hospital stay rate, increased turnover of beds and of resolutions in the care process. The objective of this study is to develop a system for bed management of the University Hospital Professor Edgard Santos - HUPES in order to monitor the patient's stay time, using the Kanban methodology as a work strategy. The method is an applied research for an agile system development process based on OpenUP, comprising four phases: design, requirements, elaboration and execution in an interactive and cyclical way, using the architectural concept of layers - MVC. Establishes color classification criteria for the elaboration of the monitoring panel - red, yellow or green, according to the Kanban methodology, calculating the days of stay of the patient in the hospital, directed by the competency and SIGTAP code, informed in the Authorization of Hospital Internment. As a result, the implementation of this method in conjunction with the system, qualified the management of care, monitoring the patients hospitalized in the care units, allowing and describing the complexity characteristics regarding length of stay, to assist in bed management and in the production of indicators of each unit. As a conclusion, it was identified the time of use of the occupied beds, evaluation of the quality of the assistance, which favors the administrative and quality indicators for the monitoring of the hospitalization process, thus providing de-hospitalization.

KEY WORDS: Universitary Hospital. Bed Management. Information System. Kanban. 


\section{INTRODUÇÃO}

A superlotação hospitalar sempre foi um grave problema no Brasil e no Mundo, causando retardo no atendimento dos pacientes agudos, aumentando com isso a mortalidade, podendo representar, inclusive, uma ameaça aos direitos humanos (HEISLER, 2012). Além disso, minimiza a qualidade da assistência, eleva os custos, a insatisfação dos usuários e profissionais, formando assim um ciclo vicioso. A solução desse problema não é pontual, pois envolve os diferentes níveis de atenção à saúde.

Uma Organização Hospitalar atende aos critérios estabelecidos pelo Sistema Único de Saúde. Isso inclui o repasse informações claras, precisas e confiáveis, mantendo o controle e medindo a eficiência dos seus indicadores de desempenho, além de permitir a demonstração de resultados do seu processo de trabalho, sempre em busca de resultados positivos.

Para isso, faz-se necessário uma gestão estratégica, que ressalte a importância de utilizar indicadores que demonstrem esses resultados com transparência (AUNSCHAU et al., 2017). Por exemplo, em uma unidade hospitalar, quando se obtém um planejamento de alta hospitalar adequado, pode-se conter custos e melhorar os resultados para os pacientes, garantindo que deixem as unidades hospitalares de maneira segura, no momento apropriado do seu tratamento, podendo até manter a continuidade do cuidado no seu pós-alta de forma organizada. $\bigcirc$ menor tempo de permanência dos pacientes reflete no maior giro de leito, aumentando a oferta de vagas hospitalares, demonstra a qualidade da equipe assistencial, indicando sua maior capacidade de resolutividade, além de contribuir com a minimização das taxas de infecção.

Porém, a avaliação também tem o seu lado negativo. Quanto maior o tempo de permanência do paciente na unidade hospitalar, maior o custo, pois cada dia de internação com valor maior do que o autorizado na tabela do SUS gera o custo do paciente-dia. E, dessa forma, haverá um reflexo não só no tempo de permanência, mas também no giro de cada leito hospitalar, tornando esse valor cada vez mais baixo, gerando déficit capaz de inviabilizar o funcionamento do hospital.

Situações como essas podem comprometer a gestão financeira do hospital, além dos seus recursos e controles internos. Portanto, faz-se necessário um controle maior da situação com reflexo real de identificação dos erros, corrigindo-os em tempo hábil para que os seus serviços atendam aos critérios estabelecidos pelo Sistema Único de Saúde.

O sistema de Informação Hospitalar SIH é o meio de faturamento hospitalar, que traduz a remuneração dos hospitais pelos serviços produzidos, cujo pagamento é feito por meio da apresentação da Autorização de Internação Hospitalar - AlH, a qual é apresentada como fatura e base de cálculo para ações da assistência de média e alta complexidade, com base na tabela do SUS (BRASIL, 2017). Porém, existe, para cada procedimento realizado nos pacientes, limites de permanência no período de internação que podem impactar financeiramente na receita hospitalar, que não recebe, muitas vezes, a compensação financeira adequada. Segundo o Ministério da Saúde (BRASIL, 2017), o tempo médio de permanência é dado pela relação entre o número de pacientes-dia e o de saídas de pacientes (altas, transferências externas e saídas por óbito) em um mesmo período.

Mesmo com a evolução tecnológica, a qualidade dos serviços de saúde prestados não tem deixado os usuários satisfeitos. Faz-se necessário levar em consideração que estamos dentro de um sistema complexo, e que é importante identificar e especificar que o valor de qualidade do serviço deve atender às perspectivas do paciente (ESSINGER, 2017). Nesse contexto, na tentativa de melhorar ou redesenhar os processos, as organizações dos serviços de saúde, assim como outras no meio 
administrativo, têm criado ferramentas para promover melhores resultados com rapidez e eficácia, sem grandes perdas financeiras.

Nessa perspectiva, a metodologia Kanban é adaptável a qualquer atividade com produção em série, sendo atualmente utilizada como processo de melhoria contínua nos mais variados segmentos das organizações hospitalares, ou seja, como um instrumento para avaliação da qualidade da assistência, para melhorar fluxos, taxas de indicadores e resolutividade dos pacientes internados, sendo uma excelente ferramenta de apoio à gestão de leitos (HEISLER, 2012). Dessa forma, permite otimizar a oferta de leitos, avaliação das necessidades de transferências, redução do índice de permanência hospitalar, o que aumenta o índice de rotatividade dos leitos e de resolutividades no processo assistencial, resultando na desospitalização (MATTOS, 2016).

O Kanban é uma expressão de origem japonesa, utilizada em empresas de fabricação de série, como, por exemplo, a Toyota Motors Company, criado na década de 50. Este método está relacionado à utilização de "cartão" ou "sinalização" com cores diferentes para indicar o andamento dos fluxos de produção desde o seu ponto inicial até $\circ$ final, indicando o status de determinadas tarefas, como "finalizado"; "em andamento"; "não executado" (SOUSA et al., 2017), tornando visíveis e disponíveis todas às informações inerentes ao processo.

O objetivo do conceito da Toyota Motors Company é criar, por meio do processo sem desperdícios e com eficiência, a maximização do valor para o cliente, que vai expandir de uma organização para culturas, indústrias e serviços diferentes (SCHIMIDT; LIPPERT; PACHECO, 2015). Pode também ser definido como um sistema de ordens de produção e compra, cujo objetivo é controlar a quantidade da produção dos produtos no momento necessário. (FERNANDES; GODINHO, 2007).

Vale ressaltar que a implementação e utilização da aplicabilidade de técnicas como
- Kanban trouxe tanto sucesso para as empresas industriais quanto para os serviços e organizações de saúde. Porém, neste caso, somente se torna efetiva com o envolvimento da equipe multidisciplinar (NEGRI; CAMPOS, 2018), principalmente os gestores das unidades assistenciais, pois a avaliação e monitoramento deverão ocorrer em um processo contínuo, com adesão participativa da equipe, durante todo período de internação do paciente, podendo implantar, aplicar e adaptar em todas as etapas do processo de forma simultânea (MATTOS, 2016). Além disso, destaca-se como potencial ferramenta para geração de resultados positivos na qualidade da assistência e organização administrativa, pois apresenta fácil acesso aos indicadores de média permanência hospitalar, além da previsão de alta.

Alguns autores como Mattos (2016) e Aguilar-Escobar, Bourque e GodinhoGallego (2015) descrevem a importância da metodologia Kanban nas organizações e serviços de saúde, com resultados e experiências bastante positivas, como alto nível de controle dos processos assistências, que além de gerar indicadores para nortear e facilitar o acompanhamento dos dados desenvolvidos. Dessa maneira, a sua aplicação nessa área inova o processo de gestão, elevando a qualidade institucional, auxiliando no processo de segurança do paciente, solucionando problemas e desenvolvendo pessoas, aproveitando o potencial de cada uma.

O estudo se desenvolve no Hospital Universitário Professor Edgard Santos (HUPES), da Universidade Federal da Bahia (UFBA). De acordo com a Unidade de Gestão e Regulação de Leitos, foi identificado que o tempo de uso dos leito $s$ ocupados está acima do que foi pactuado em contratualização hospitalar, necessitando de um melhor monitoramento do processo de internação, de forma a proporcionar a desospitalização segura.

Desse modo, o objetivo deste estudo é desenvolver um sistema para gestão de 
leitos do Hospital Universitário Professor Edgard Santos (HUPES), a fim de monitorar o tempo de permanência do paciente, utilizando a metodologia Kanban como estratégia de trabalho. Como justificativa, como apontado por Hendy et al. (2013), o fato de o número de leitos disponível para a população estar diminuindo, o que demanda atenção especial aos existentes para que se consiga, por meio deles, melhores resultados, seja do ponto de vista assistencial, seja financeiro.
Portanto, esta pesquisa sobre a capacidade do sistema possibilitará a disponibilidade das informações em tempo real, facilitando a visualização e acesso para equipe multiprofissional. Isso proporcionará a melhoria do processo decisório, otimização da oferta e o aumento do índice de rotatividade do leito e a redução da média de permanência hospitalar.

\section{PROCEDIMENTO METODOLÓGICO}

O percurso para construção do método inicia-se com a classificação da pesquisa. Segundo Turrioni e Mello (2012), é um estudo de natureza aplicada a uma pesquisa exploratória e descritiva. Sua abordagem é de caráter combinado e utiliza o método baseado na pesquisa-ação.

O estudo constitui-se de uma pesquisa metodológica aplicada ou tecnológica, especificada devido ao processo de desenvolvimento e criação de um novo produto (ANGELONI et al., 2016). Neste caso, um software web, de aplicação prática, voltado para a solução do problema de otimização da gestão de leitos, considerando para isso o tempo de permanência do paciente e agilidade no processo de decisão.

A pesquisa foi desenvolvida no Hospital Universitário Professor Edgard Santos, terciário, de alta complexidade, localizado no município de Salvador - Bahia. O método de desenvolvimento utilizado, OpenUP, foi definido em quatro fases interativas e cíclicas: concepção, requisitos, elaboração e execução (MANCERA-ARANGO, 2017). A Figura 1 apresenta o fluxograma multifuncional da metodologia. 


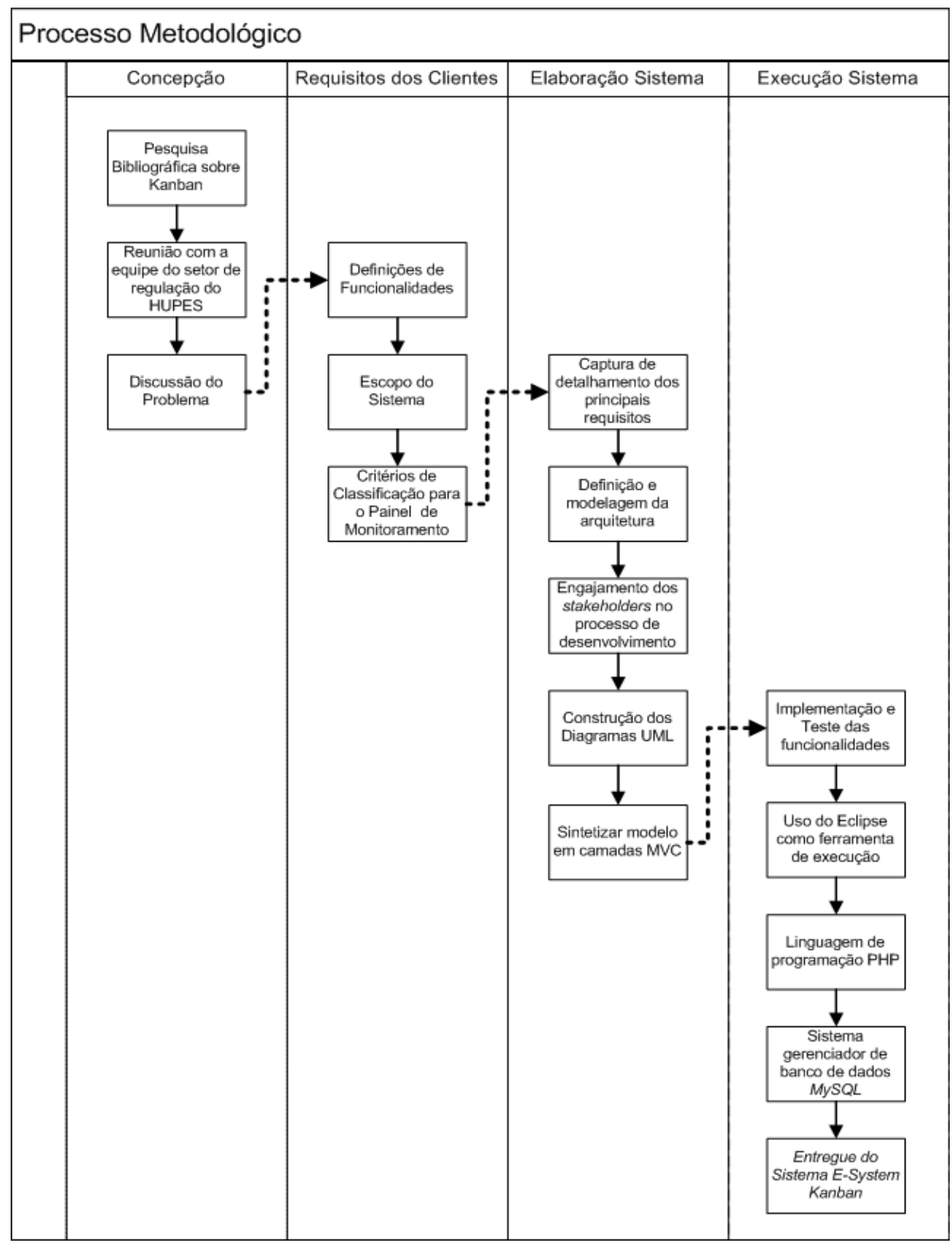

Figura 1 - Fluxograma multifuncional do processo metodológico.

Fonte: Dados dos autores (2019).

Inicialmente, na fase de concepção, realizou-se uma pesquisa bibliográfica sobre a metodologia Kanban. Da mesma forma, foram realizadas reuniões entre as equipes da unidade de Gestão e Regulação de Leitos e Setor de Gestão de Processos e Tecnologia da Informação do HUPES para melhor compreensão do domínio do problema e entendimento simultâneo entre todos os interessados.

Para a construção dos requisitos, foram identificadas as funcionalidades-chaves, definindo o escopo do sistema. Os stakeholders relataram os dados dos pacientes necessários para inserção no sistema, por meio da interface. Além disso, ficaram estabelecidos os critérios de classificação por cores para a elaboração do Painel de Monitoramento - verde, amarelo ou vermelho, conforme a metodologia Kanban, sendo o verde, dentro do intervalo entre o número de dias mínimo permitidos; amarelo, dentro do intervalo entre o número de dias da metade do tempo ou igual ao total de dias; e o vermelho, maior que o número de dias permitido.

O sistema calcula os dias de permanência do paciente na internação, conforme 
competência e código SIGTAP informado na Autorização de Internamento Hospitalar - AlH. Desse modo o sistema irá contabilizar o tempo de permanência conforme estabelecidos os critérios de classificação por cores, demonstrado no quadro 1.

\begin{tabular}{|c|l|l|l|}
\hline \multicolumn{2}{|c|}{ CLASSIFICAÇÃO PARA O SISTEMA ELETRÔNICO KANBAN } \\
\hline VERDE & $\begin{array}{l}\text { PERMANÊNCIA } \\
\text { IDEAL }\end{array}$ & $\begin{array}{l}\text { Dentro do intervalo entre } \\
\text { o número de dias mínimo, } \\
\text { permitidos pela tabela SIGTAP. }\end{array}$ & $\begin{array}{l}\text { Conforme exemplo = de } \mathbf{3} \\
\text { a dias }\end{array}$ \\
\hline AMARELO & $\begin{array}{l}\text { PERMANÊNCIA } \\
\text { ALERTA }\end{array}$ & $\begin{array}{l}\text { Dentro do intervalo entre o } \\
\text { número de dias da metade do } \\
\text { tempo ou igual ao total de dias, } \\
\text { permitidos pela tabela SIGTAP. }\end{array}$ & $\begin{array}{l}\text { Conforme exemplo }=\text { de } \mathbf{3} \\
\text { a dias. }\end{array}$ \\
\hline VERMELHO & $\begin{array}{l}\text { PERMANÊNCIA } \\
\text { ULTRAPASSADA }\end{array}$ & $\begin{array}{l}\text { Maior que o número de dias } \\
\text { permitido pela tabela SIGTAP. }\end{array}$ & $\begin{array}{l}\text { Conforme exemplo }= \\
\text { maior que } \mathbf{6} \text { dias. }\end{array}$ \\
\hline
\end{tabular}

Quadro 1 - Critérios para classificação de cores para o sistema eletrônico.

Fonte: Dados dos autores (2019).

A figura 2 apresenta o Sistema de Gerenciamento unificado de procedimentos, medicamentos e órteses/próteses e materiais de síntese do SUS - SIGTAP/DATASUS. Utiliza como exemplo um paciente internado para um procedimento que tem atributo de média de permanência para 6 dias.

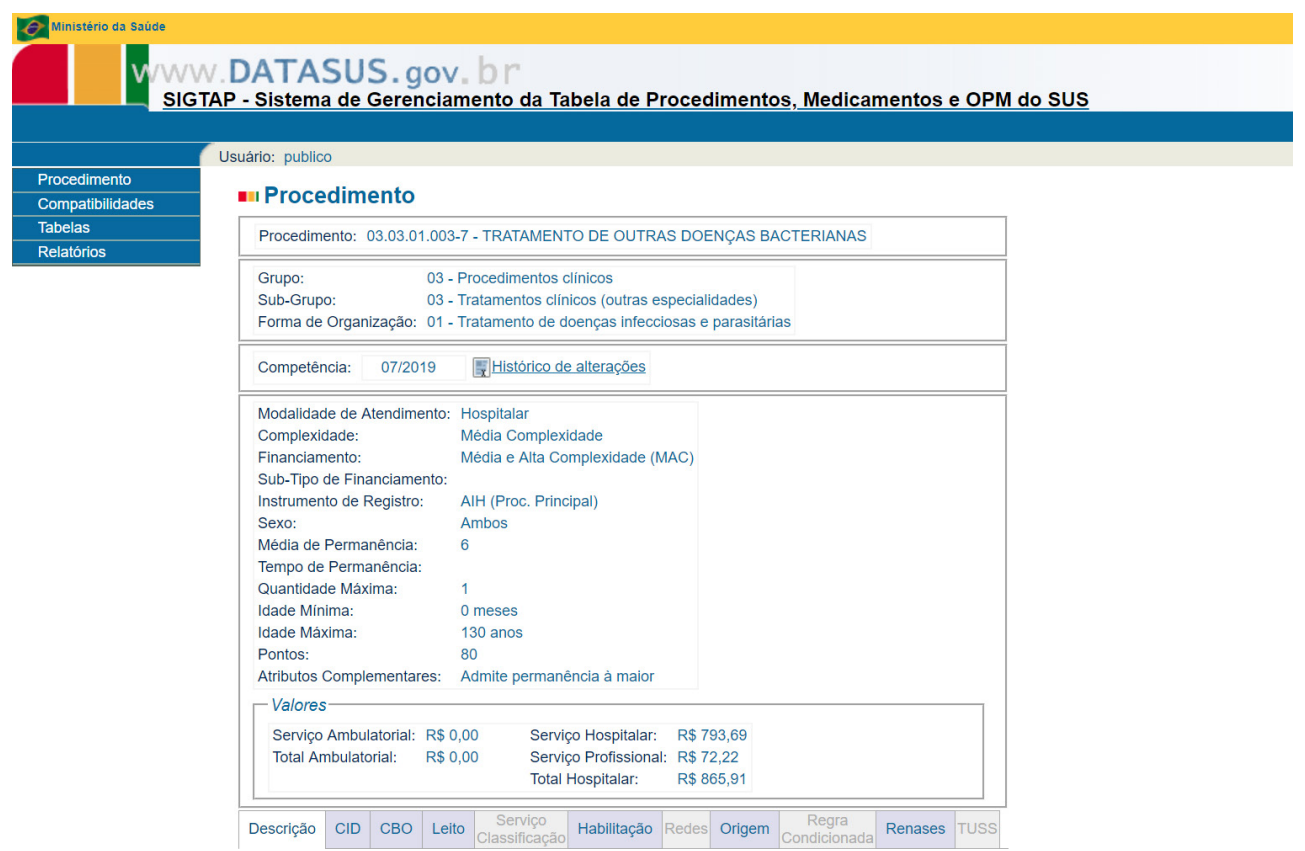

Figura 2 - Sistema de gerenciamento de procedimentos.

Fonte: SIGTAP (2018).

Diante da proposta descrita acima, o sistema eletrônico Kanban foi construído em formato de painel de monitoramento, de forma transparente e de fácil acesso para todos os profissionais envolvidos na assistência, para que se possa monitorar e avaliar as condições e o tempo de permanência do paciente na unidade conforme intervalo de dias e as cores definidas na metodologia utilizada, além de visualizar a previsão de alta, diante do tempo de internação preconizado por cada código de procedimento. A figura 3 exemplifica, no painel de monitoramento, o tempo de permanência e status de cada paciente da unidade. 

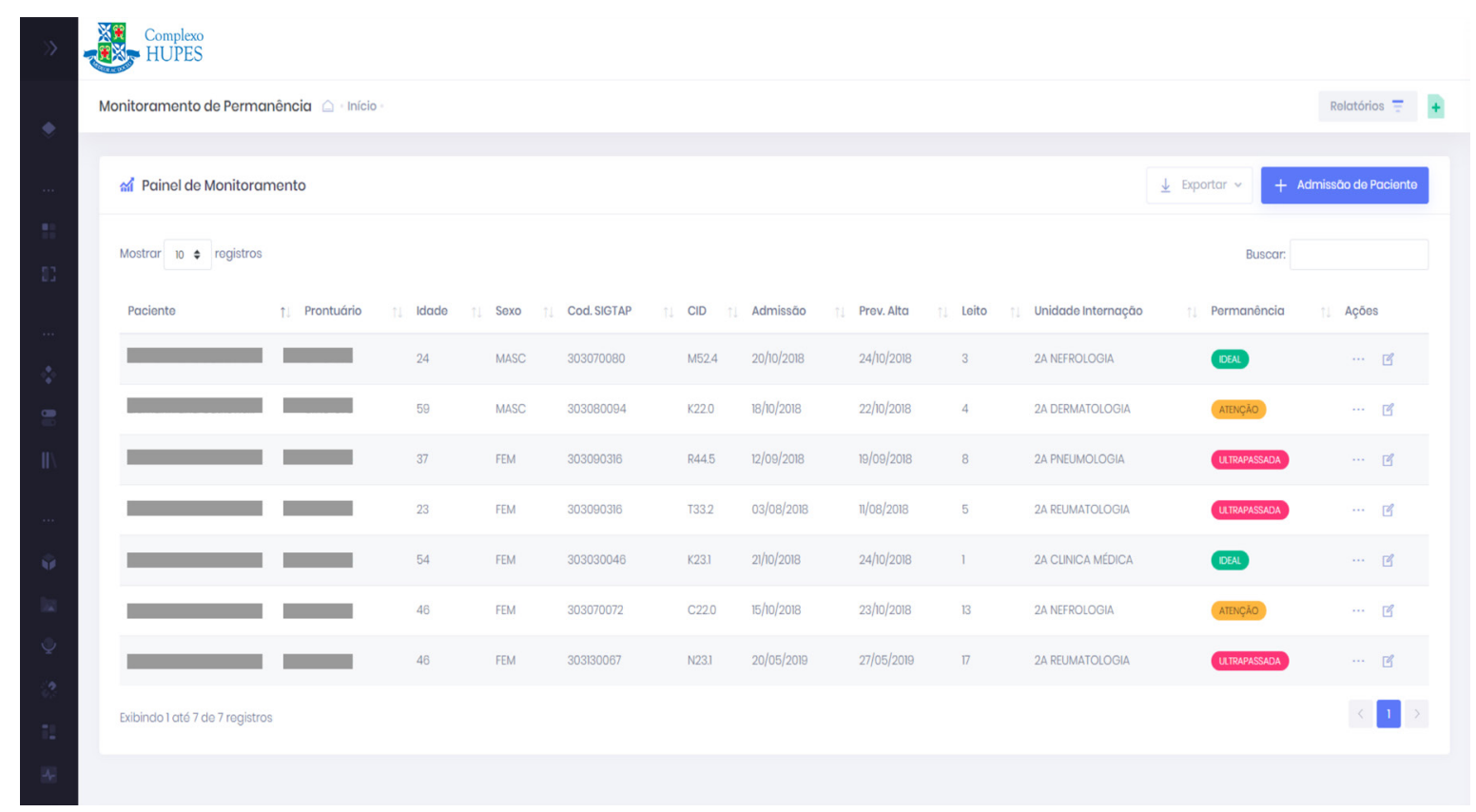

Exibindo 1 tó 7 do 7 rogistros

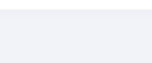

Figura 3 - Painel de monitoramento.

Fonte: Dados dos autores (2019).

$\mathrm{Na}$ fase de Elaboração, os principais objetivos foram: a captura de detalhamento dos principais requisitos, a definição e modelagem da arquitetura e a obtenção de maior engajamento dos stakeholders no processo de desenvolvimento. Dessa forma, foi modelada a arquitetura do software e construídos os diagramas UML (Unified Modeling Language), de casos de uso, de atividades e de classes, utilizando o software AstahCommunity.

A essência da descrição arquitetural sintetizou-se ao modelo em camadas MVC (Model-View-Controller), contendo algumas decisões iniciais importantes que definem a estrutura do projeto conforme Figura 4. A escolha desse padrão deu-se pelo benefício de separar as regras de negócios da lógica de apresentação e da interface com - usuário, possibilitando alterações nas interfaces sem a necessidade de modificações nas regras de negócios. Assim, proporciona a flexibilidade, facilidade na manutenção e reutilização de códigos.

A comunicação entre a interface e o banco de dados é definida por meio de um controlador (controller), tornando possível a separação em camadas bem definidas. Dessa maneira, quando um usuário executa um comando na interface gráfica, como um clique em um botão, a interface irá se comunicar com o controlador que, por sua vez, comunica-se com as regras de negócios (model) e responde ao usuário por meio da interface gráfica do navegador (view). A figura 4 abaixo demonstra a entrada do usuário, o processamento e a resposta visual para o usuário, os quais são separados e gerenciados por camadas distintas. 


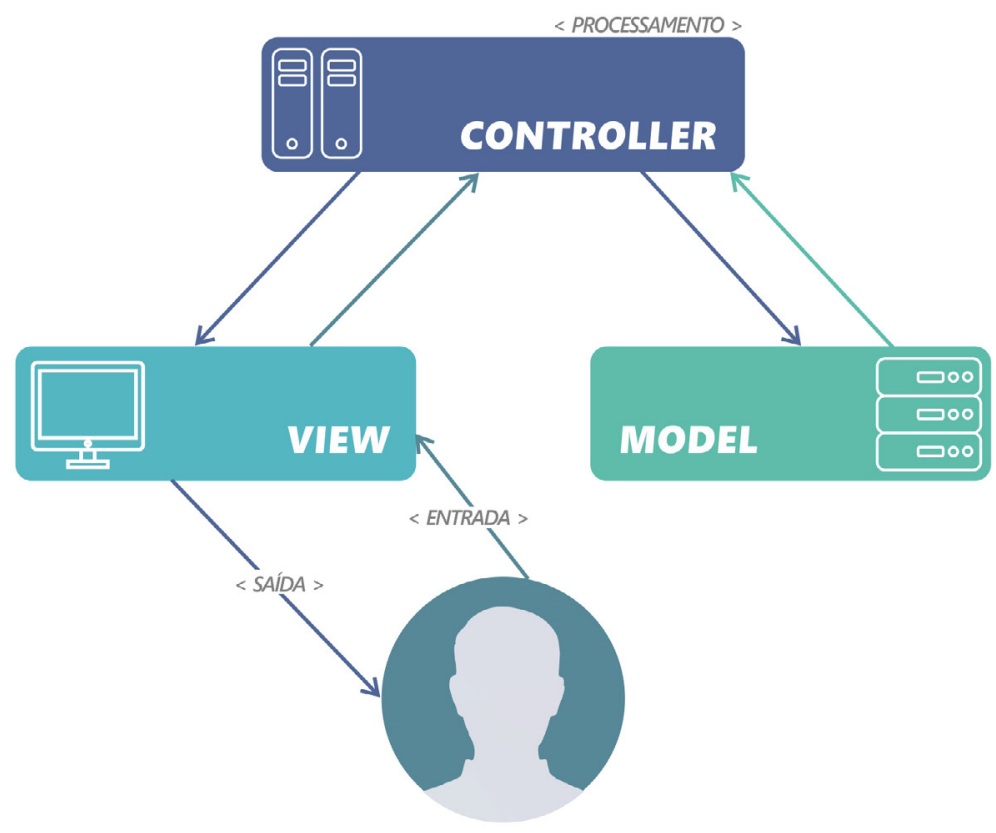

Figura 4 - Arquitetura MVC.

Fonte: Dados dos autores (2019).

Já na fase de Execução, iniciou-se a implementação e teste das funcionalidades. Para isso, foram utilizadas as seguintes ferramentas: o Eclipse IDE, a linguagem de programação PHP e o sistema gerenciador de banco de dados MySOL (COWBURN, 2018). Nessa etapa,

o sistema eletrônico Kanban foi entregue, conforme apresentado na figura 3.

\section{RESULTADO E DISCUSSÕES}

Indicadores são medidas usadas para ajudar a descrever a situação de um determinado fenômeno ou problema, fazer comparações, verificar mudanças ou tendências e avaliar as ações planejadas durante um período de tempo, em termo de qualidade e quantidade das ações executadas (KLUCK et al., 2002).

Dando ênfase a necessidade de conhecer todos os problemas relacionados à superlotação dos serviços de saúde e qual a melhor estratégia para minimizar esse problema que, automaticamente e em cascata, trará melhorias nos indicadores de tempo de permanência, giro de leito e taxa de ocupação hospitalar. Assim, o sistema eletrônico Kanban, como metodologia estratégica, faz parte de um conjunto de ferramentas utilizados para transformar e aperfeiçoar constantemente na tentativa de resolver os problemas elencados, atendendo a demanda real e existente para acompanhamento da situação, com criatividade e inteligência.

$\mathrm{Na}$ definição do uso do sistema eletrônico Kanban, antes da instalação nas unidades escolhidas, a discussão de grande relevância foi a importância do apoio da alta gestão, o preparo do ambiente em que o sistema será implantado, e o envolvimento ativo, em todas as fases do processo, de todos os níveis hierárquicos do hospital. Depois de conceituada a metodologia e implantado o sistema, é preciso que todos colaboradores executem sua parte no momento em que a atividade está sendo 
avaliada. O sucesso produtivo e administrativo é interdependente, ou seja, está vinculado à participação de todos, pois é preciso executar a atividade no momento esperado, desencadeando a necessidade da próxima atividade, e assim por diante.

Diante disso, foram realizadas reuniões estratégicas com as equipes envolvidas, esclarecendo sobre a proposta do sistema, conceitos sobre a metodologia Kanban, sua aplicação, funcionalidades e objetivo. A partir disso, foi criada uma planilha simples
Microsoft Excel $\cap$, enviada semanalmente para as unidades, por e-mail, pela Unidade de Gestão e Regulação de Leitos, mostrando a taxa de ocupação das unidades e o tempo permanência de cada paciente, solicitando a justificativa do tempo elevado em relação à resposta a esse e-mail. A sua finalidade era aproximar a proposta do sistema e conhecimento dos gestores sobre o tempo dos pacientes nas suas unidades. A figura 5 , demonstra o exemplo da planilha Excel®, criada conforme descrição acima.

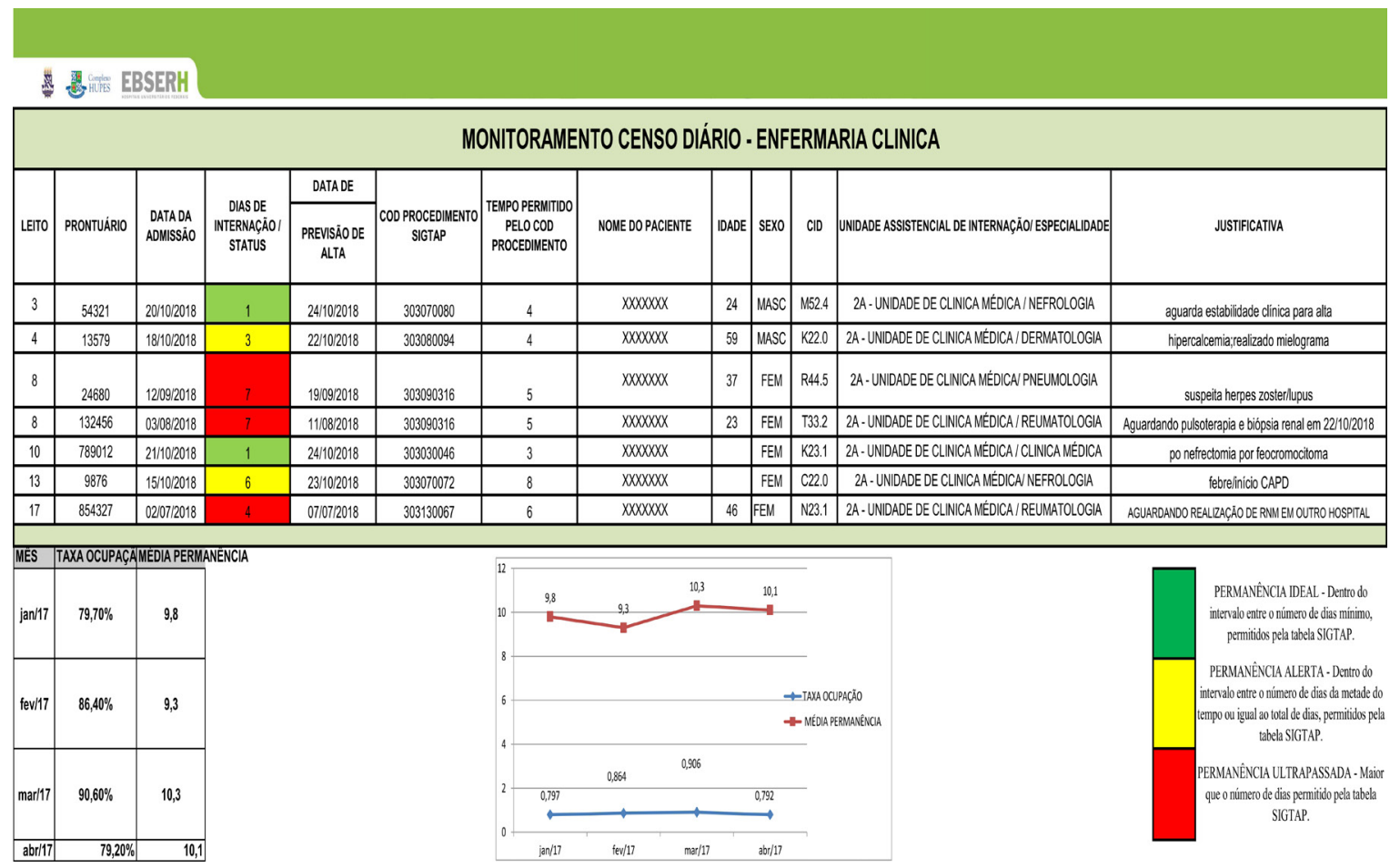

Figura 5 - Planilha Excel: Dados característicos dos pacientes com avaliação de tempo de internação em leitos clínicos. Fonte: Dados dos autores (2019).

Além das reuniões, foram necessários treinamentos, organizados em conjunto com a Divisão de Gestão de Pessoas e Educação Permanente do Hospital, tendo como público principal a equipe médica de residentes, inicialmente, permitindo a participação de todos sem interferência no horário de trabalho, utilizando para isso os intervalos das visitas de equipe nas próprias unidades. Também foram discutidos os processos e necessidades de gerenciamento do sistema junto à equipe de enfermagem que está presente nas ações e discussões sobre as condutas necessárias para implementações dos cuidados com o paciente $24 \mathrm{~h}$ por dia. Diante disso, o sistema foi implantado como proposta piloto em duas unidades do hospital do estudo: uma unidade de clínica médica e outra unidade de clínica cirúrgica, caracterizadas pelos diferentes perfis dos pacientes e acordos realizados com os gestores médicos e de enfermagem, como facilitadores dos processos de trabalho.

A aplicabilidade da metodologia Kanban, associada ao sistema eletrônico, mostrou-se viável para o controle, monitoramento e análise da média de permanência 
na instituição, sinalizada pelos parâmetros da tabela unificada de procedimentos medicamentos e órteses próteses, e materiais de síntese do Sistema Único de Saúde - SIGTAP/DATASUS. Dessa forma, fortaleceu o processo de tomada de decisões no âmbito financeiro, correlacionados à data de admissão do paciente, calculando a previsão de alta.

O sistema eletrônico Kanban simboliza os registros pelas três cores definidas (verde, amarelo e vermelho), considerando o tempo de permanência dos pacientes pela data de admissão, traduzindo as respostas, condutas a serem adotadas e ações mitigadoras em casos de necessidade de correção. A sua atualização é feita de forma automática, pois o sistema exporta os dados pessoais do paciente no momento da admissão, do sistema matriz do hospital para o sistema eletrônico Kanban. A partir desse momento o tempo de permanência já é computado, mantendo o monitoramento do período de internação desse paciente, com a data previsão de alta, baseado no código de procedimento descrito na $\mathrm{AlH}$.

O monitoramento e avaliação dos dados registrados no sistema Kanban são realizadas diariamente pela equipe multiprofissional das unidades assistenciais e Unidade de Gestão e Regulação de Leitos, ao longo de todo curso da hospitalização do paciente. Os médicos assistentes de cada paciente sinalizam à enfermeira da unidade os problemas encontrados que impactam no tratamento do paciente, e que podem prolongar a sua permanência, procedendo com as devidas correções e ajustes para a tomada de decisão.

A equipe de monitoramento é responsável por analisar e controlar a permanência do paciente, agindo diretamente na decisão do médico responsável, que realiza a justificativa do paciente de estar na situação de "alerta ou ultrapassada", o que é identificado no painel com as cores amarela ou vermelha, ou seja, não obteve alta. A situação é avaliada e direcionada para a possibilidade de resolução, evitando prolongamento do tempo de permanência do paciente na unidade hospitalar.

Caso a situação não seja solucionada, após avaliação e análise dos problemas encontrados e que dificultam a alta desse paciente, é direcionada para a alta gestão do hospital. Em segunda instância, este setor redireciona para a Superintendência, com a descrição do motivo da longa permanência e da não resolutividade, a fim de que seja definida a conduta a ser adotada.

Os principais indicadores para gestão hospitalar, na unidade do estudo, demonstraram acentuados processos evolutivos, os quais foram comparados desde o ano de 2016 a 2018, cujas taxas de ocupação evoluíram de 70,4\% para 79,8\%; e a média de permanência hospitalar baixou de 9,38 para $6,1 /$ dias, conforme demonstrativo na figura 6. Vale ressaltar que essa melhoria ocorreu quando se iniciaram as avaliações para implantação do sistema.
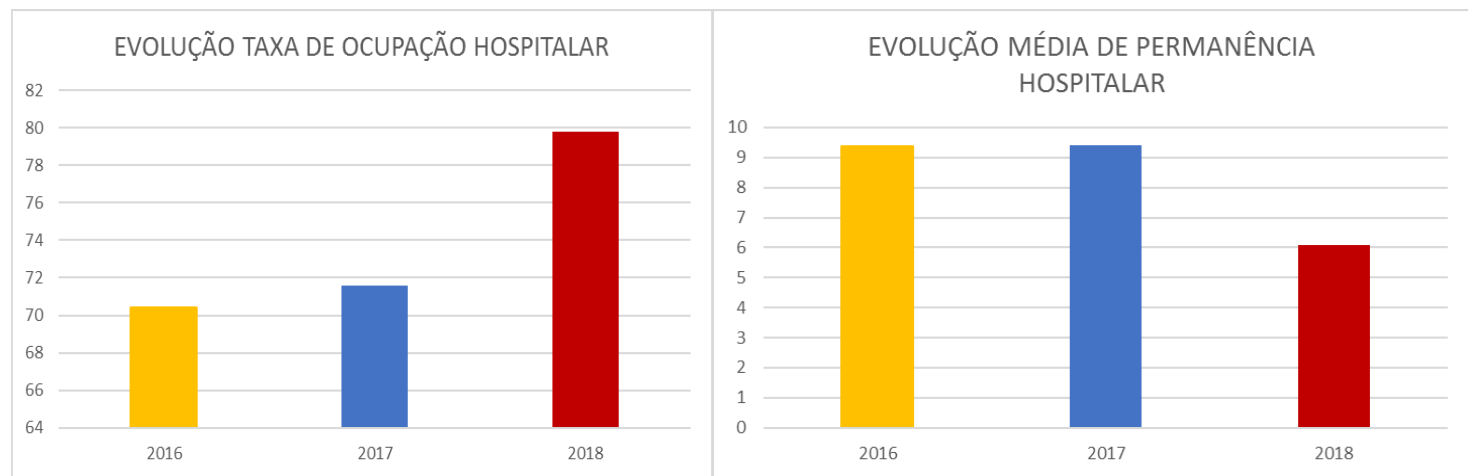

Figura 6 - Gráfico de evolução da taxa de ocupação hospitalar e média de permanência dos anos de 2016 a 2018. Fonte: Baseado em SMART/Gestão à vista - Intranet HUPES. 
Vale destacar também que o Kanban serviu com uma grande ferramenta de gestão da clínica, pois, além de todos os benefícios alcançadas no aumento da taxa de ocupação e minimização do tempo de permanência, foi possível aumentar o quantitativo de chamadas de pacientes em lista de espera, girando ainda mais o leito e minimizando a morbimortalidade de pacientes com patologias tão complexas. Na figura 7, abaixo, demonstramos o crescimento mensal do total de pacientes internados no ano de 2018, até o mês de setembro, em leitos clínicos.

\section{TOTAL DE PACIENTES EM LISTA DE ESPERA INTERNADOS EM 2018 POR MÊS}

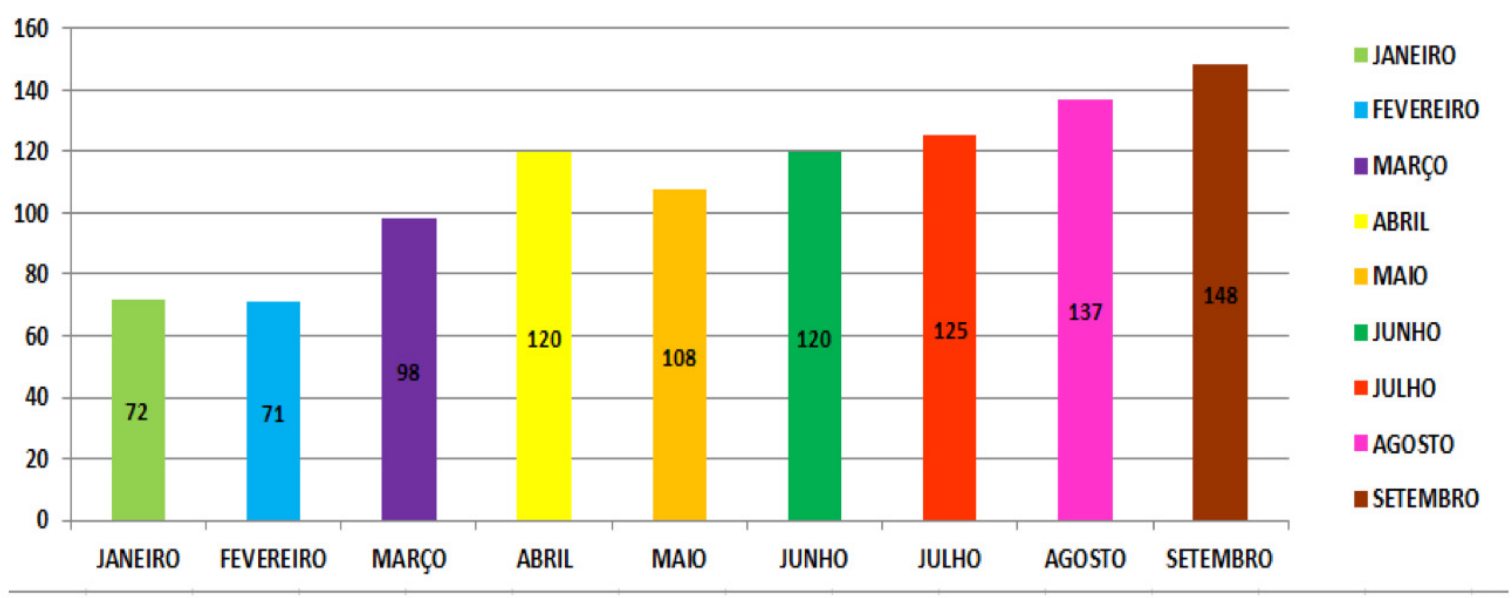

Figura 7 - Total de pacientes internados por mês em 2018 - Internação em leitos clínicos.

Fonte: Dados dos autores (2019).

Nenhuma dessas conquistas foi possível sem o comprometimento da equipe multidisciplinar, que teve extrema importância para a adequada implantação do sistema eletrônico Kanban nas demais unidades assistenciais. Esses indicadores serão os responsáveis por fornecer não somente à gestão, mas a todos os envolvidos, informações estratégicas para serem traçados processos de melhoria e fluxos de desospitalização segura e satisfatória para os pacientes.

Essa metodologia, quando ativa e participativa, favorece processos de caráter educativo, uma vez que permite o planejamento de ações estratégicas para atender a necessidade do tratamento proposto ao paciente no momento da sua admissão. Dessa forma, proporciona, por exemplo, a construção do perfil hospitalar baseada no levantamento das patologias incidentes, confecção de uma avaliação baseada no tempo de internação do paciente por patologia, com discriminação de cada categorização por dia, conforme Sistema de Internação Hospitalar - SIH/ SUS (BRASIL, 2017).

Com isso, a facilidade da aplicação da metodologia Kanban, por meio do sistema eletrônico, proporcionou o monitoramento diário de cada unidade assistencial pelas equipes, que analisaram o tempo médio de permanência dos seus pacientes. Esses profissionais observaram, então, a melhora do fluxo de internamento do paciente no hospital, rotatividade dos leitos de internação, o que proporcionou maior interação da equipe multiprofissional na definição de condutas e qualidade do atendimento dos pacientes, minimizando assim os custos hospitalares. 


\section{CONSIDERAÇÕES FINAIS}

Considerando o objetivo geral deste estudo, ou seja, desenvolver um sistema para gestão de leitos do Hospital Universitário Professor Edgard Santos - HUPES, a fim de monitorar o tempo de permanência do paciente, utilizando a metodologia Kanban como estratégia de trabalho, faz-se necessário alinhar os aspectos mais marcantes neste campo de atuação.

O trabalho iniciado pela equipe da Unidade de Gestão e Regulação de Leitos do HUPES com a ferramenta Kanban, já tem a sua extrema importância para o monitoramento dos indicadores hospitalares. Hoje, a unidade possui o mapa de leitos com a identificação de todos os pacientes internados no hospital, com demonstração dos dados em tempo real, caracterizando a data de admissão, previsão de alta e tempo de permanência, possibilitando maior controle e identificação das ações facilitadores junto às equipes da unidade para promoção da desospitalização segura.

Após operacionalização, observamos um aumento considerável dos internamentos clínicos para 36\%, quando comparado primeiro como o segundo quadrimestre de 2018, além de minimizar o tempo de espera de três meses para quinze dias. Esse dado é importante, pois antes do monitoramento dos leitos e tempo de permanência dos pacientes, pacientes "moradores" permaneciam nas unidades assistenciais do hospital, o que prejudicava a possibilidade de outros pacientes ocuparem os leitos em tempo hábil. Esse foi o impacto de maior positividade, pois foi possível viabilizar os internamentos, por exemplo, de especialidades complexas e de referências para - Estado como a gastro-hepatologia e onco-hematologia, cujo pacientes são de maior morbimortalidade.

Em primeiro lugar, a metodologia promove a verificação da aplicabilidade do sistema, nas unidades piloto de escolha, para que seja um facilitador, conseguindo dessa forma dinamizar o trabalho da equipe multiprofissional e, junto com a equipe da Unidade de Gestão e Regulação de Leitos, desospitalizar, utilizando os recursos de forma racional, segura e organizada. Possibilitará, obviamente, a minimização da alta permanência, avançando em melhorias, como a minimização das infecções e custos hospitalares, e aumento do giro de leito, segurança do paciente, entre outros ganhos.

Em segundo lugar, e de extrema importância, permite melhorias no processo de comunicação entre as equipes multidisciplinares, a Unidade de Gestão e Regulação de Leitos e as unidades assistenciais hospitalares. Consequentemente, proporciona maior facilidade no conhecimento dos problemas de cada paciente, direcionados a um objetivo comum: a desospitalização segura.

Posteriormente, o sistema eletrônico Kanban será instalado em todos os demais computadores das outras unidades assistenciais no hospital do estudo, facilitando o acesso à equipe multiprofissional da visualização de todos os pacientes internados. Essa facilitação será feita por meio da sinalização por cores do seu status, conforme a classificação do Kanban, correspondente ao tempo de permanência do paciente, e para aqueles que estejam fora do planejamento desse tempo, o desenvolvimento das ações para a resolução do problema.

Algumas limitações podem ser apontadas neste estudo para que futuramente possam pontuar processos de melhoria. A literatura é muito direcionada para a estratégia do modelo Kanban como processo de avaliação e apoio nas indústrias. Alguns artigos encontrados abordam essa estratégia de gestão hospitalar em poucas áreas, principalmente no que trata de unidades de urgência e emergência, apesar de ser uma forma bem adaptada pra a proposta do modelo. Porém, foi observado que a implantação da ferramenta como processo de gestão assistencial apresenta dificuldades na adesão das equipes e acompanhamento, 
pois a falta de conhecimento dos processos administrativos dificulta a gestão assistencial.

Ademais, foi observado, por exemplo, grande dificuldade em realizar modelos comparativos com outras unidades hospitalares desde o inicio do processo de implantação de indicadores de monitoramento construídos até os resultados alcançados. Diante dos obstáculos encontrados, este artigo servirá como facilitador para o processo de gestão hospitalar direcionado para a gestão de leitos, tornando o trabalho entre equipes viável, visível e valorizado, dando início à possibilidade do direcionamento de dados e indicadores comparativos internos para posteriormente servir como comparativo entre outras unidades hospitalares.

Contudo, pode-se concluir que a metodologia Kanban é relevante, importante e traz inúmeros benefícios na otimização dos resultados, gerando indicadores que atendem os objetivos, entre eles as melhorias contínuas na performance da execução do controle de permanência hospitalar. Consequentemente, gera a redução de custos, alinhamento da comunicação entre as unidades essenciais para monitoramento dos dados, satisfação dos usuários da gestão e das unidades assistenciais que utilizam esse conceito. Cabe salientar ainda a importância da manutenção do plano estratégico com o cronograma e treinamento para que haja engajamento no seu sucesso contínuo e permanente. 


\section{REFERÊNCIAS}

AGUILAR-ESCOBAR, V. G.; BOURQUE, S.; GODINO-GALLEGO, N. Hospital Kanban system implementation: Evaluating satisfaction os Nursing personnel. Investigaciones Europeas de Direccion y Economia de la Empresa, v. 21, n. 3, p. 101-110, 2015.

ANGELONI, M. T. et al. Gestão da informação e do conhecimento em projetos de pesquisa e desenvolvimento: um estudo de caso. In: SIMPÓSIO INTERNACIONAL DE GESTÃO DE PROJETOS, INOVAÇÃO E SUSTENTABILIDADE, 4., 2016, São Paulo. Anais [...]. São Paulo, 2016.

AUNSCHAU, F. et al. Avaliação de Intervenção da Gestão da Clínica na Qualificação do Cuidado e na oferta de Leitos em um Hospital Público de Grande Porte. Revista Scientia Medica, v. 27, n. 2, 2017.

BRASIL. Ministério da Saúde. Secretaria de Atenção à Saúde. Sistema de |Informação Hospitalar: Manual Técnico Operacional do Sistema. 2017. Disponível em: http://www.saude. sp.gov.br/resources/ses/perfil/gestor/homepage/auditoria/ manuais/manual_sih_janeiro_2017.pdf. Acesso em: 29 ago. 2019.

COWBURN, P. PHP Documentation Group. Disponível em: http://php.net/. Acesso em: 5 out. 2018.

ESSINGER, Luiz Alexandre. Implantação da Ferramenta Kanban como indutora de Melhora do Fluxo dos Pacientes em um Hospital de Emergência. 2017. 67 f. Dissertação (Mestrado Profissional em Medicina, área de concentração Técnicas Videoendoscopicas) - Universidade Federal do Rio de Janeiro, Rio de Janeiro, 2017.

FERNANDES, F. C. F.; GODINHO, F. M. Sistemas de coordenação de ordens: revisão, classificação, funcionamento e aplicabilidade.

Revista Gestão \&Produção, São Carlos, v. 14, n. 2, 2007.

\section{HEISLER, P. A. Aplicação da metodologia Kanban como} ferramenta adaptada para gestão de "leitos" na emergência. 2012. 25 f. Trabalho de Conclusão de Curso (Especialização em Informação Científica e Tecnológica em Saúde) - Instituto de Comunicação e Informação Científica e Tecnológica em Saúde, Fundação Oswaldo Cruz, Porto Alegre, 2012. Disponível em: http://www.arca.fiocruz.br/handle/icict/6505. Acesso em: 6 out. 2018. 
HENDY, P. et al. In-Depth Analysis of Delays to Patient Discharge: a metropolitan teaching hospital experience. Journal of the

Royal College of Physicians, v. 12, n. 4, p. 320-323, 2013.

KLUCK, M. et al. A gestão da qualidade assistencial do Hospital de Clínica de Porto Alegre: implantação e validação de indicadores. Revista de Administração em Saúde, São Paulo, v. 4, n. 16, p. 27-32, 2002.

MANCERA-ARANGO, G. K. Sistema para lavisualización en mapas de indicadores epidemiológicos de tuberculosis para la Secretaría de Salud de Manizales. Revista Ingenieria Solidaria, v. 13, n. 22, p. 1-21, 2017.

MATTOS, Corina Maria. Aplicação do Kanban ao processo de internação de uma unidade hospitalar pediátrica. 206. $108 \mathrm{f}$. Dissertações (Mestrado profissional em Enfermagem Assistencial) - Universidade Federal Fluminense, Niterói, RJ, 2016.

NEGRI, S. C.; CAMPOS, M. D. O Uso da Ferramenta Kanban para o Controle da Permanência dos usuários SUS. Convibra Administração. Disponível em: www.convibra.com.br/dwp. asp? $i d=5191 \&$ ev=25. Acesso em: 5 out. 2018.

REGNER, A. P.; NASCIMENTO, R. I. M. Enfrentamento da Superlotação da Emergência do HNSC: oportunizando a discussão sistêmica do modelo de atenção do HNSC. Porto Alegre, RS: Gerência de Pacientes Externos/Grupo Hospitalar Conceição, 2011.

SCHIMIDT, W. C.; LIPPERT; M.; PACHECO, D. A. J. Análise da Implantação do Sistema Kanban no Processo de Injeção de Plásticos. Revista Eletrônica da Faculdade de Ciências exatas e Agrárias: Produção, Construção e Tecnologia, v. 4, n. 6, 2015.

SILVA, A. O. et al. Metodologia Kanban. 2015. Disponível em: https://www.webartigos.com/artigos/metodologiakanban/138389 Acesso em: 22 nov. 2018.

SISTEMA DE GERENCIAMENTO DA TABELA DE PROCEDIMENTOS, MEDICAMENTOS E OPM DO SUS - SIGTAP. 2018. Disponível em: http://sigtap.datasus.gov.br/tabelaunificada/app/sec/inicio.jsp Acesso em: 3 maio 2019.

SOUSA, P. R. et al. Gestão do Fluxo de Pacientes em Internações Relacionadas ao Pronto Socorro: aplicação da Metodologia

Kanban. Revista de Inovação Hospitalar e Inovação em Saúde, Belo Horizonte, v. 14, n. 1, jan./mar. 2017. 
STENDER, Gustavo Henrique Cordeiro. Lean Health Care:

Modelo de Implantação de Ferramenta Kanban a um

almoxarifado de um Hospital Federal no Rio de Janeiro. 2016. 66

f. Dissertação (Mestrado em Engenharia da Produção) - Centro

Federal de Educação Tecnológica Celso Suckow da Fonseca, Rio de Janeiro, 2016.

TURRIONI, J.; MELLO, C. Metodologia de pesquisa em

engenharia de produção: estratégias, métodos e técnicas para condução de pesquisas quantitativas e qualitativas. Itajubá:

UNIFEI, 2012. 